\title{
Comprehensive Immunohistochemistry: Digital, Analytical and Integrated
}

\author{
Arvydas Laurinavicius $^{a, b}$ Benoit Plancoulaine ${ }^{c}$ Paulette Herlin ${ }^{a}$ \\ Aida Laurinaviciene ${ }^{a, b}$ \\ ${ }^{a}$ Department of Pathology, Forensic Medicine and Pharmacology, Faculty of Medicine, Vilnius University, \\ and ${ }^{b}$ National Center of Pathology, Affiliate of Vilnius University Hospital Santariskiu Clinics, Vilnius, Lithuania; \\ 'Path-Image, Inserm (UMR 1199), Unicaen, University of Normandy, Cancer Center F. Baclesse, Caen, France
}

\section{Key Words}

Digital image analysis · Digital pathology .

Immunohistochemistry · Proteomics · Tumor heterogeneity

\begin{abstract}
Immunohistochemistry (IHC) is widely used in contemporary pathology as a diagnostic and, increasingly, as a prognostic and predictive tool. The main value of the method today comes from a sensitive and specific detection of a protein of interest in the context of tissue architecture and cell populations. One of the major limitations of conventional $\mathrm{IHC}$ is related to the fact that the results are usually obtained by visual qualitative or semiquantitative evaluation. While this is sufficient for diagnostic purposes, measurement of prognostic and predictive biomarkers requires better accuracy and reproducibility. Also, objective evaluation of the spatial heterogeneity of biomarker expression as well as the development of combined/integrated biomarkers are in great demand. On the other end of the scale, the rapid development of tissue proteomics accounting for 2D spatial aspects has led to a disruptive concept of next-generation IHC, promising high multiplexing and broad dynamic range quantitative/spatial data on tissue protein expression. This 'evolutionary gap' between conventional and next-generation IHC can be filled by comprehensive IHC based on digital tech-
\end{abstract}

nologies (empowered by quantification and spatial and multiparametric analytics) and integrated into the pathology workflow and information systems. In this paper, we share our perspectives on a comprehensive IHC road map as a multistep development process.

(c) 2016 S. Karger AG, Basel

\section{Introduction}

The last decade was marked by rapid development of disruptive technology enabling high-resolution scanning of whole-slide imaging (WSI) and leading to the concept of digital pathology. Since the nature of microscopic image - the very object of a pathologist's special expertise - is switching from analogous to digital systems, many aspects of the specialty will transform. This change involves a vast spectrum of new ways of storing, transmitting, processing and analyzing the data and information used in pathology diagnosis and research [1-4]. Broadly speaking, combining laboratory and clinical information systems with new options of digital imaging and decision support in pathology will allow pathologists to act in a distributed environment of integrated multidisciplinary patient care, evolving into the 'cloud pathology' way of work [5]. In addition, digital pathology becomes an im-

\section{KARGER}

E-Mail karger@karger.com

www.karger.com/pat
(C) 2016 S. Karger AG, Basel

$1015-2008 / 16 / 0833-0156 \$ 39.50 / 0$
Arvydas Laurinavicius

National Center of Pathology, Affiliate of Vilnius University Hospital Santariskiu Clinics P. Baublio 5

LT-08406 Vilnius (Lithuania)

E-Mail arvydas.laurinavicius@vpc.lt 
portant chain in the pipeline of tissue biomarker research and drug development, supporting biobanking, (pre-) clinical studies and integromics, in general [6].

One of the major advantages of the WSI technology relates to the fact that enormous amounts of tissue pathology data are retrieved in digital format and available for computational processing [7, 8]. Augmented by countless combinations of visualization, illumination and computational modalities, the opportunities of image analysis-based applications for tissue pathology appear infinite. One might expect that 'evolutionary pressure' will select the tools with the highest added value for personalized medicine. However, to translate them into clinical routine, comprehensive validation of the technology for diagnostic purposes across the complete spectrum of surgical pathology represents the most important barrier [8]. Another, less obvious but important impediment is lack of dissemination and collaboration between biomedical and computational investigators: often, a pathobiological application is presented as a proof of concept rather than a sophisticated investigative study, while pathology communications are sketchy about the physical/ engineering side of the work [9]. Therefore, it is important to recognize and synergize the steps of the technology adoption on the way to clinical application.

Immunohistochemistry (IHC) has become a routine test in contemporary pathology to visualize specific proteins in tissue sections. It has major advantages: it enables to characterize cells and other tissue components based on their protein expression in the context of tissue architecture. Visual interpretation of the IHC slides is usually based on qualitative and semiquantitative evaluation of the chromogenic IHC reaction product along with the tissue morphology; since hundreds of different proteins can be detected based on their antigenicity, this method is of great value in the diagnosis of tumors and other pathologies. Being relatively inexpensive, available in many laboratories, and usually doable on formalin-fixed paraffinembedded tissues, IHC became 'another special stain', which, in many occasions, has replaced histochemistry and electron microscopy. Consequently, during the four decades of IHC use in tumor diagnosis, pathologists have acquired some bad habits, essentially when judging the IHC result via the perception of a 'good' stain that 'pleases the eye' [10]. Meanwhile, the complexity of the IHC method and its vulnerability to multiple factors of variation in (pre)analytical phases have long been recognized and addressed with the concept of 'the total IHC test', advocating that the performing laboratory should assume responsibility for all steps of the IHC procedure [11].
Since then, great progress has been made in standardization and automation of the IHC staining procedures along with the efforts to better control the preanalytical phase and to improve visual scoring/counting systems of the IHC results for various tasks. In this current state of the technology, conventional IHC is a powerful tool for pathology diagnosis; however, it has inherent limitations to serve the needs of personalized therapies, which increasingly rely on quantitative biomarker measurement.

Personalized therapies require robust stratification of the patients into prognostic and predictive categories based on reliable information of various nature: clinical, radiologic, pathologic and molecular testing, for example. Often, multiple testing modalities have to be combined for optimal results. Protein biomarkers, measured in the diseased tissue, are important since they are actionable molecules that represent the end effect of genetic abnormalities and pathway disarrangements, and may become targets of therapy. The need for quantitative IHC as a tool for quantitative protein biomarker assessment in the tissue or in situ proteomics is well acknowledged; various modalities, options and limitations have recently been reviewed by Taylor [10] and Carvajal-Hausdorf et al. [12]. The scope of issues and opportunities on the way to the quantitative IHC is really broad, just to outline the main:

1 The preanalytical phase of the IHC test, which can be critical for protein detection, is hard to standardize and control

2 A true reference standard to calibrate and control the analytic accuracy of the test is hard to achieve in IHC; a quantitative internal reference standard based on double IHC to compare the quantity of an intrinsic tissue protein seems the most practical option

3 Bright-field chromogenic IHC presents the tissue architecture and is preferred by pathologists, but immunofluorescence-based techniques are better suited for multiplex antigen detection and true quantification; these alternatives remain to be considered for specific tasks, since IHC does not provide data on the true concentrations of proteins

4 Proper quantification of the IHC signal is only possible with the use of whole slide digital image analysis (DIA) with its own multiple modalities and specific needs for validation and standardization

The concepts of quantitative IHC and digital IHC seem now to be used interchangeably to convey both the aim and the new technology available to optimize information retrieval from the IHC test. The quantitative IHC reflects the quest for converting IHC to a tissue-based test resembling ELISA or quantitative in situ proteomics [10]. 
Fig. 1. Road map for comprehensive IHC. A multistep process with key components is outlined. It represents an open framework rather than a complete list of tasks and opportunities.

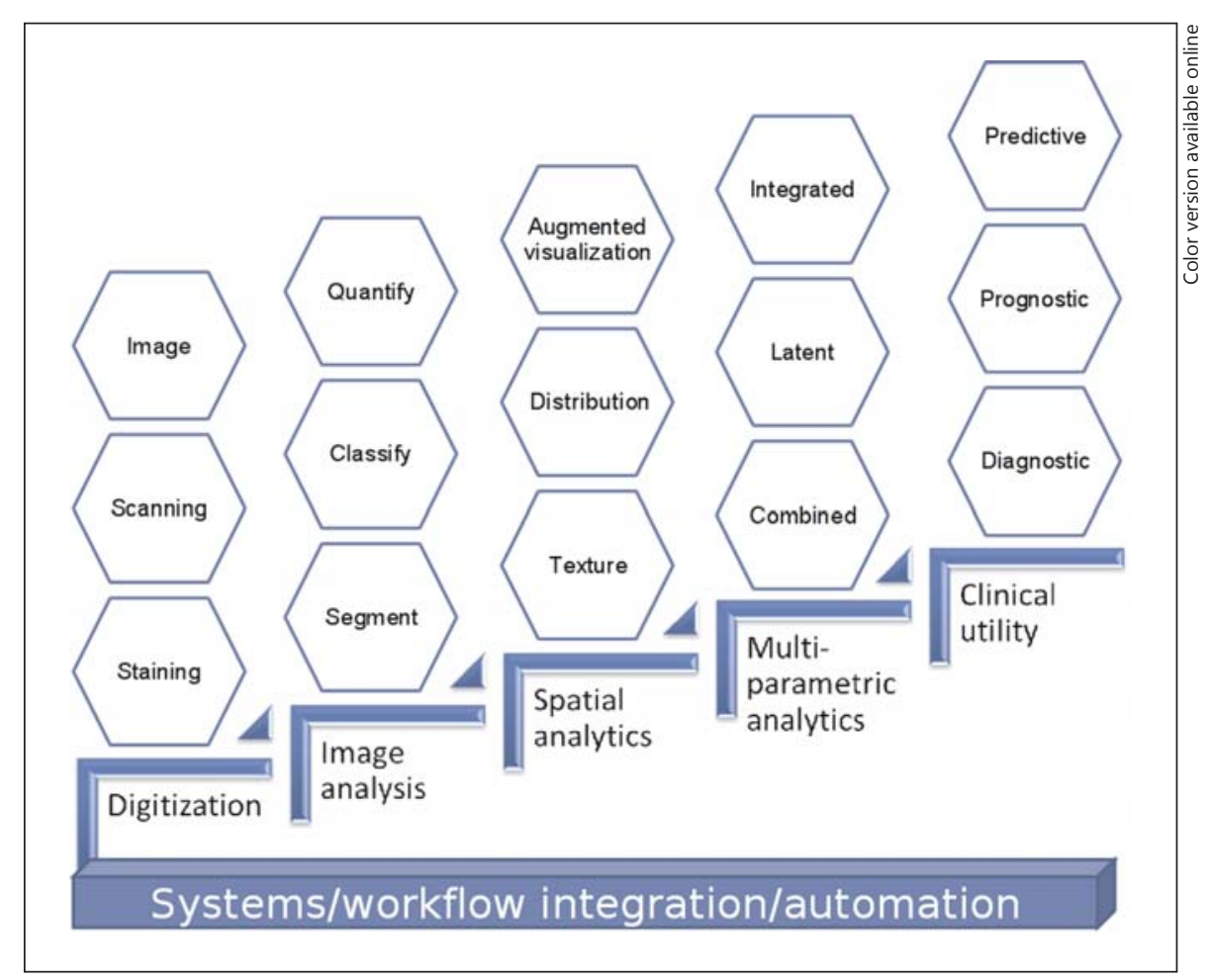

However, IHC brings a significant advantage over these techniques: the signal can be measured and interpreted in the context of 2D biological tissue structures. With advanced DIA and computation opportunities, methodologies for spatial analytics can and should be developed to produce comprehensive data sets, combined/multiparametric indicators and augmented visualization of the tissue protein biomarkers. Therefore, we suggest that the term analytical IHC better reflects this broader scope of benefits. Starting from the total IHC test concept, empowered by digital technologies, appropriate analytics, and data and workflow integration, the test can evolve into comprehensive IHC, which is adopted in the clinical and research environment. Of course, the future may bring other technologies into the ecosystem of ' $2 \mathrm{D}$ tissue proteomics'. In particular, new techniques based on a combination of mass spectroscopy with IHC for highly multiplex, directly quantitative and broad dynamic range tissue proteomics have already been proposed and introduced as next-generation IHC [13-15].

The terminology and semantics is not the primary topic of this essay. The multiple terms proposed (and yet to come) reflect various perspectives on the vast field of opportunities opened by the disruptive WSI technologies, where the physical world of the total IHC test meets the digital universe of virtually unlimited computational modalities. Accordingly, teams of researchers at this interface and the research community in general need to develop a common language and understanding. The road map will evolve eventually to address various steps and aspects. Meanwhile, one should refrain from high expectations of 'quick fixes' and clinical utility to be proven for every new tool or methodology at the stage of 'proof of principle'. Evolution of comprehensive IHC can be viewed in the context of bioimage informatics, which is still in its early stage of development; as Gene Myers noted: 'From my perspective, it is very reminiscent of the state of bioinformatics in the early 1980s: the exciting, somewhat chaotic free-for-all that is potentially the birth of something new' [16].

\section{Road Map for Comprehensive IHC}

We envision the road map of the comprehensive IHC as a multistep process, reliant on the total IHC test concept, starting with digitization of the IHC slides, then image processing and analysis, computation of appropriate analytics, and resulting in unique clinical benefits for individual patients (fig. 1). Various clinical tasks may re- 
quire different constellations of the steps and components, and adjustment to specific biomarkers, tissues and clinical outcomes, too. Every step would require appropriate validation and quality assurance (QA) procedures; on the other hand, digital processing may serve QA needs of the total IHC test, e.g. by monitoring staining intensity. Importantly, successful implementation of digital pathology solutions in laboratory information systems and workflow will require a sound basis. As a matter of fact, the road map represents main driving forces and barriers for clinical adoption: clinical benefits, obtained from unique and reproducible information and reinforced by efficient integration into the patient care systems, have to outweigh the costs of additional qualified staffing, infrastructure, image analysis, and computational and QA procedures.

Various aspects of quantification of protein biomarkers by IHC have been reviewed recently $[10,12,17]$. In the many studies published so far, reproducibility and capacity advantages of IHC image analysis were used to quantify protein and/or protein-expressing cell populations to predict clinical outcomes. Indeed, in most studies, image analysis-based quantification has been shown useful and was not inferior to pathologists' scoring [18]. Some solutions have been validated for clinical use. However, while validated tests and therapies are considered clinically useful, usefulness should not become a substitute for accuracy or objectivity [19]. An intrinsic obstacle exists to fully apply the rules of analytic test validation [20] for IHC: accuracy of the method can be estimated only if reference standards (criterion standard, ground truth) are available, which is difficult to achieve in IHC. Validation of the tests is usually performed by comparing digital results with manual estimates, either quantitative or semi-quantitative, or by comparing image analysis results with another form of reference standard, e.g. fluorescence in situ hybridization or clinical data [18]. Although these approaches are common and useful, reference standards in these studies are indirect and may be subject to their own bias. Ideally, to validate and calibrate quantitative IHC tests, one should seek the most direct reference standard that answers the same question as the test is intended to do [21]. While reference standards for protein quantification by IHC can be achieved by histones, quantitative internal reference standards based on double IHC or other novel techniques $[10,12]$, it is much more complicated to generate reference standards and controls for the detection of cells and tissue structures by image analysis. Since, in addition to variations in tissue processing, significant variance in the morphology of diseased tissue is unavoidable whereas protein quantification by IHC is expected to be performed in the context of tissue structures, efficient solutions for this aspect of validation present a challenge. For example, tumors frequently present with very variable cell morphology, cellularity, growth patterns and staining properties; thus, the very object of IHC image analysis is inherently variable and impacts the levels of accuracy that could be achieved. The processing of very large WSIs is another problem, as it is difficult to refine cell and tissue detection and identification using sophisticated but timeconsuming algorithms.

We have proposed a methodology to validate and calibrate DIA of Ki67 IHC in breast cancer tissue based on a reference standard obtained from the same WSI by counting positive and negative tumor cell profiles using a stereology test grid of systematically sampled frames [22]. The approach enabled to measure the bias of DIA and visual evaluation from five pathologists who participated in the study. Furthermore, the DIA measurement accuracy was improved after calibration and measurement error correction steps based on the quantified bias. This procedure allowed to decrease patient misclassification by the DIA Ki67 cutoffs of 10, 15 and 20\% down to 5-7\%, compared to that of the visual evaluation consensus (the median) of five pathologists at $11-18 \%$. Although the methodology was successful and could be applied in clinical studies, its broader application for internal laboratory validation and QA procedures would be limited due to the dependence on expert human resource to produce the reference data. This limitation has been recognized in bioimage informatics stating that full-scale adoption of automated DIA tools would require efficient production and maintenance of the reference data sets and provision of integrated editing tools [23]. The amount of manual expert work needed on large images with a huge number of cells is a critical bottleneck in the adoption of editingbased approaches [24]. Several approaches have been proposed to decrease the expert workload [25-27]; however, the efficiency and integration of the tools requires further sophistication.

Quantification of specific biomarkers by digital IHC for clinical needs is the topic most frequently addressed by the studies; however, more unique aspects of digital IHC tests, namely, spatial and multivariate analytics, can be a real 'gold mine'. Indeed, digital IHC opens the opportunity to quantify a biomarker expression in various tissue compartments and to obtain its distribution and texture indicators, which may bring benefits compensating for some of the quantification accuracy limitations discussed above. 


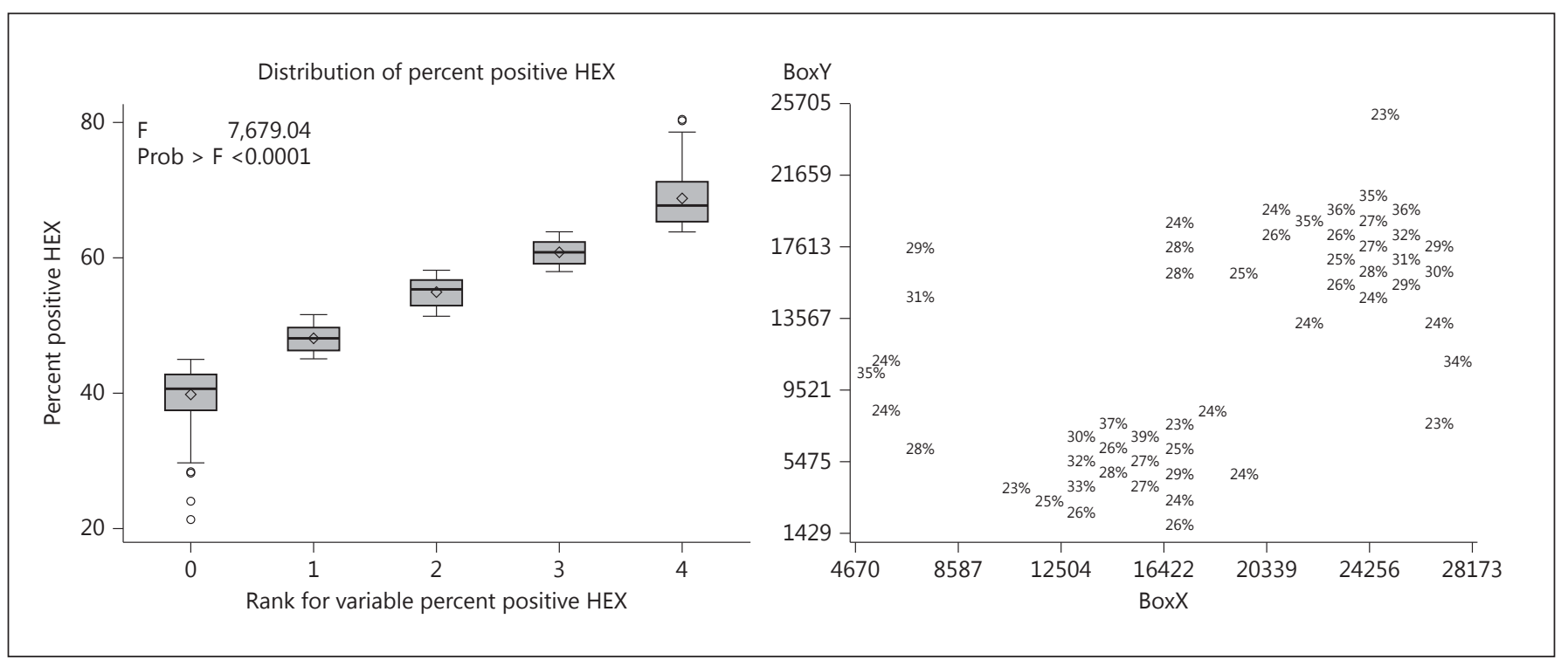

Fig. 2. Pareto hot spot: mapping of spatial distribution of the upper quintile of IHC biomarker expression in tumor tissue. On the left: box and whisker plots of the quintiles of biomarker expression tested by ANOVA. On the right: the upper quintile values plotted against the $\mathrm{x}$ - and $\mathrm{y}$-coordinates of the WSI.

\section{Spatial (2D) IHC for Tissue Microenvironment Assessment}

Assessment of the spatial aspects is firstly needed in tumor pathology where the phenomenon of intratumor tissue heterogeneity is recognized as a serious obstacle in biomarker measurement and use for companion diagnostics. Furthermore, spatial heterogeneity assessment may retrieve unique information on tumor biology, reflecting interactions between distinct subpopulations of tumor and nontumor cells. Heindl et al. [28] have recently reviewed the progress in digital pathology to map spatial heterogeneity in the tumor microenvironment: various texture- and density-based spatial clustering methods have been employed to uncover visually undetectable spatial cell interactions in tumors. They proposed to categorize spatial analysis techniques into four types of methods: (1) spatially defined tumor areas; (2) measurement of spatial distances; (3) statistical methods including graphs and clustering, and (4) spatial statistics.

Spatial statistics is a powerful method used for many years in geography, ecology and other fields advanced in spatial modeling. Among the various techniques, spatial statistics derived from regular polygons in arrays have been shown to be most efficient for mapping spatial variation and to provide the most common framework for spatially explicit models $[29,30]$. However, to the best of our knowledge, these approaches have been underexplored in digital pathology [31]. We have recently conducted a feasibility study [32] to measure the Ki67 labeling index in breast cancer tissue, taking intratumor heterogeneity of the biomarker expression into account. Hexagonal tiling of the Ki67 IHC DIA data enabled to combine average Ki67 positivity per tumor with a rich set of distribution and texture indicators. Importantly, the data allowed multiple definitions of the expression of biomarker hot spots (based on percentiles); for example, the upper quintile of the hottest tumor area represents proliferative activity in roughly $20 \%$ of the tumor cell population and could be defined as 'Pareto hot spot' (fig. 2). Furthermore, the data allowed augmented visualization of the hot spots and proliferative surface (texture) of the tumors. Of note, the concept of augmented visualization emerges as the ability to extract quantitative data from images and present these back to the pathologist working visually, providing the ability to 'see' tissue characteristics that would normally escape the naked eye [6]. Last but not least, hexagon tiling of DIA data simulates systematic subsampling of the data or multiple measurements of the tissue and could thus be used as a QA measure by automated highlighting of outliers and potential analysis artifacts. 


\section{Multiparametric IHC for Integrated Biomarkers}

Besides the spatial analytics, DIA of IHC slides has another important advantage compared to the visual evaluation: it generates continuous data of broad dynamic range in a high-capacity manner. This enables their use for multivariate statistic methods, which are much more powerful than those used in studies based on visual assessment. This is a fundamental advantage because cancer is a highly heterogeneous disease which can be truly understood and managed in a multidimensional space of the biological hallmarks of cancer [33]. Accordingly, molecular profiling of cancer requires multiple biomarkers analyzed by multivariate methods. For example, multiparametric measurement strategies have been employed by the multigene classifiers used in breast cancer to complement traditional pathology methods; however, it is possible that more robust and simpler methods based on IHC could provide comparable information [34]. Cuzick et al. [35] have proposed the IHC4 score based on a combination of four IHC markers, commonly used in breast cancer, and suggested that the amount of prognostic information provided by the IHC4 was similar to that in the mRNA-based, 21-gene Genomic Health recurrence score. Subsequently, clinical utility of the IHC4 score, supplemented with clinicopathologic parameters (IHC4+C score) [36] or by antiapoptotic protein BAG1 measurement by IHC [37], was reported. In general, the combinatorial approach to IHC-based testing has been rather extensively explored for prognostic stratification of breast cancer patients [38]; however, the combined IHC biomarkers proposed were based on visual qualitative or semiquantitative evaluation.

We have previously demonstrated the feasibility to obtain multivariate IHC characteristics of breast tumor tissue based on DIA of a set of 10 IHC markers on tissue microarrays [39]. Factor analysis of the data uncovered latent interdependencies in the IHC profiles which could not be identified by simple pairwise statistics. In particular, we found that a major factor of aggressive disease behavior, associated with histological grade and relevant intrinsic subtypes of breast cancer, was characterized by opposite loadings of hormone receptors/BCL2 and Ki67/ HIF-1 $\alpha$. The second major factor of variation was represented by predominant SATB1 and HIF-1 $\alpha$ expression; however, this factor was not associated with any clinicopathologic parameters in the study. Remarkably, in our recent study of the same patient cohort, supplemented with data on disease outcome, we found this latter factor to be an independent predictor of overall survival of the

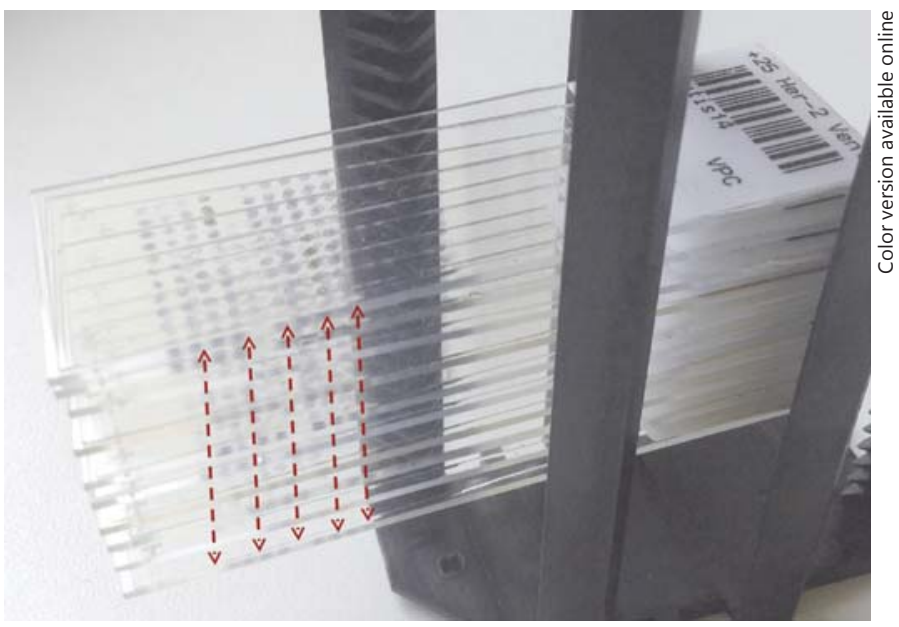

Fig. 3. Multiparametric IHC. Consecutive sections of tissue microarrays stained for 10 different IHC biomarkers and processed by DIA allow multivariate analysis of tumor immunophenotypes.

patients [40]. This demonstrates that combined or integrated prognostic factors can be discovered from the multiparametric IHC approach in 'an evidence-based manner'.

In these studies, we were able to retrieve the 'multidimensional' tumor immunophenotype information from automated DIA data of 10 IHC biomarkers stained on consecutive sections of tissue microarrays (fig. 3). This surrogate (indirect) multiplex IHC, empowered by the digital technologies and multivariate statistics, was sufficient to retrieve useful information. Again, the multidimensionality of the integrated biomarkers may compensate for relative inaccuracies of a single IHC biomarker measurement; this trend is also illustrated by a recent study applying 3 different IHC biomarkers to measure proliferative activity of breast cancer and testing prognostic cutoffs for each of them [41]. One might expect that true multiplex IHC techniques, made available for pathology laboratories [42], would supply a powerful tool in the framework of comprehensive IHC.

\section{Integration and Automation}

It is difficult to overemphasize the importance of efficient integration of digital pathology systems into the clinical and biomarker research environments. While specific applications for specific tasks can be used as stand-alone tools or services 'on top' of clinical routine, broad adoption of new tools is highly dependent on prop- 
er integration and automation of the systems and processes. The vectors of integration can seem unlimited in the era of big data and integromics. Comprehensive IHC is not an exception and can serve as an example of possible integration needs and benefits, firstly, to facilitate individualized patient-centered health care and increase the efficiency of care providers.

Implementation of a comprehensive IHC platform to the 'conventional' or 'digital' pathology workflow adds processes which might be quite different from existing ones; requirements for setting up a digital pathology core facility have been discussed in detail [6]. Nevertheless, a digital pathology platform is expected to follow general standards of pathology laboratories, specimen tracking, quality control, turnaround time monitoring and lean management, for example. From this perspective, comprehensive IHC could be seen as 'digital extension' of the existing IHC laboratory. On the one hand, digital technologies can improve and further automate conventional IHC processes. For example, robust DIA in IHC will require more standardized procedures of IHC staining and tissue processing [10]; on the other hand, being more sensitive to image quality variation, DIA can provide automated monitoring of IHC staining quality for the laboratory. We performed routine Ki67 IHC staining of consecutive sections of multiple tissues to analyze slideto-slide variation using DIA [43]. Importantly, this process involved integration of laboratory information systems, images and statistical analysis tools and allowed a high level of workflow automation. Factor analysis and plot visualization were performed to explore slide-toslide variation in the staining results in the control tissue. The data enabled to monitor staining parameters over time and to explore potential reasons for variation. We found that, even in consecutive serial tissue sections, tissue-related factors mainly affected the IHC DIA results. While this approach can be useful for QA of conventional IHC, our findings also indicate the need for more standardized tissue controls to be used for quantitative IHC, as noted above [10].

\section{Conclusion}

Comprehensive IHC is able to enhance the total IHC test concept via the new opportunities provided by digital pathology. It also offers a framework to better understand the challenges and opportunities. A variety of fit-for-purpose applications will evolve; however, finding the right balance of unique benefits, costs, and efficiencies can make it a pioneering area of digital pathology adoption.

\section{Acknowledgment}

This research is funded by the European Social Fund under the Global Grant measure (grant No. VP1-3.1-SMM-07-K-03-051).

\section{References}

1 Gu J, Ogilvie RW (eds): Virtual Microscopy and Virtual Slides in Teaching, Diagnosis and Research. Advances in Pathology, Microscopy and Molecular Morphology; Gu J, Hacker GW (ser eds). Boca Raton, Taylor \& Francis, 2005.

2 Soenksen D: Digital pathology at the crossroads of major health care trends: corporate innovation as an engine for change. Arch Pathol Lab Med 2009;133:555-559.

-3 Weinstein RS, Graham AR, Richter LC, Barker GP, Krupinski EA, Lopez AM, Erps KA, Bhattacharyya AK, Yagi Y, Gilbertson JR: Overview of telepathology, virtual microscopy, and whole slide imaging: prospects for the future. Hum Pathol 2009;40:1057-1069.

4 Pantanowitz L: Digital images and the future of digital pathology. J Pathol Inform 2010;1:15.

5 Laurinavicius A, Raslavicus P: Consequences of 'going digital' for pathology professionals - entering the cloud. Stud Health Technol Inform 2012;179:62-67.
6 Hamilton PW, Bankhead P, Wang Y, Hutchinson R, Kieran D, McArt DG, James J, Salto-Tellez M: Digital pathology and image analysis in tissue biomarker research. Methods 2014;70:59-73.

7 Kayser K: Quantification of virtual slides: approaches to analysis of content-based image information. J Pathol Inform 2011;2:2.

-8 Ghaznavi F, Evans A, Madabhushi A, Feldman M: Digital imaging in pathology: wholeslide imaging and beyond. Annu Rev Pathol 2013;8:331-359.

-9 Ramamurthy B, Coffman FD, Cohen S: A perspective on digital and computational pathology. J Pathol Inform 2015;6:29.

10 Taylor CR: Quantitative in situ proteomics: a proposed pathway for quantification of immunohistochemistry at the light-microscopic level. Cell Tissue Res 2015;360:109-120.

11 Taylor CR: The total test approach to standardization of immunohistochemistry. Arch Pathol Lab Med 2000;124:945-951.
2 Carvajal-Hausdorf DE, Schalper KA, Neumeister VM, Rimm DL: Quantitative measurement of cancer tissue biomarkers in the lab and in the clinic. Lab Invest 2015;95:385396.

13 Rimm DL: Next-gen immunohistochemistry. Nat Methods 2014;11:381-383.

14 Giesen C, Wang HA, Schapiro D, Zivanovic N, Jacobs A, Hattendorf B, Schuffler PJ, Grolimund D, Buhmann JM, Brandt S, Varga Z, Wild PJ, Gunther D, Bodenmiller B: Highly multiplexed imaging of tumor tissues with subcellular resolution by mass cytometry. Nat Methods 2014;11:417-422.

15 Angelo M, Bendall SC, Finck R, Hale MB, Hitzman C, Borowsky AD, Levenson RM, Lowe JB, Liu SD, Zhao S, Natkunam Y, Nolan GP: Multiplexed ion beam imaging of human breast tumors. Nat Med 2014;20:436-442.

16 Myers G: Why bioimage informatics matters. Nat Methods 2012;9:659-660. 
17 Di Cataldo S, Ficarra E, Macii E: Computeraided techniques for chromogenic immunohistochemistry: status and directions. Comput Biol Med 2012;42:1012-1025.

18 Riber-Hansen R, Vainer B, Steiniche T: Digital image analysis: a review of reproducibility, stability and basic requirements for optima results. APMIS 2012;120:276-289.

19 Tadrous PJ: On the concept of objectivity in digital image analysis in pathology. Pathology 2010;42:207-211.

20 Bland JM, Altman DG: Measuring agreement in method comparison studies. Stat Methods Med Res 1999;8:135-160.

$>21$ Laurinavicius A, Laurinaviciene A, Dasevicius D, Elie N, Plancoulaine B, Bor C, Herlin $\mathrm{P}$ : Digital image analysis in pathology: benefits and obligation. Anal Cell Pathol (Amst) 2012;35:75-78.

$\checkmark 22$ Laurinavicius A, Plancoulaine B, Laurinaviciene A, Herlin P, Meskauskas R, Baltrusaityte I, Besusparis J, Dasevicius D, Elie N, Iqbal Y, Bor C, Ellis IO: A methodology to ensure and improve accuracy of Ki67 labelling index estimation by automated digital image analysis in breast cancer tissue. Breast Cancer Res 2014;16:R35.

23 Ground-truth data cannot do it alone. Nat Methods 2011;8:885.

24 Al-Kofahi Y, Lassoued W, Lee W, Roysam B: Improved automatic detection and segmentation of cell nuclei in histopathology images. IEEE Trans Biomed Eng 2010;57:841-852.

-25 Bjornsson CS, Lin G, Al-Kofahi Y, Narayanaswamy A, Smith KL, Shain W, Roysam B: Associative image analysis: a method for automated quantification of 3D multi-parameter images of brain tissue. J Neurosci Methods 2008;170:165-178.

26 Plancoulaine B, Laurinaviciene A, Meskauskas R, Baltrusaityte I, Besusparis J, Herlin P, Laurinavicius A: Digital immunohistochemistry wizard: image analysis-assisted stereology tool to produce reference data set for calibration and quality control. Diagn Pathol 2014;9(suppl 1):S8.
27 Luisi J, Narayanaswamy A, Galbreath Z, Roysam B: The FARSIGHT trace editor: an open source tool for 3-D inspection and efficient pattern analysis aided editing of automated neuronal reconstructions. Neuroinformatics 2011;9:305-315.

28 Heindl A, Nawaz S, Yuan Y: Mapping spatial heterogeneity in the tumor microenvironment: a new era for digital pathology. Lab Invest 2015;95:377-384.

29 Birch CPD, Oom SP, Beecham JA: Rectangular and hexagonal grids used for observation, experiment and simulation in ecology. Ecol Model 2007;206:347-359.

30 Nelson TA: Trends in spatial statistics. Prof Geogr 2012;64:83-94.

31 Sharifi-Salamatian V, de Roquancourt A, Rigaut JP: Breast carcinoma, intratumor heterogeneity and histological grading, using geostatistics. Anal Cell Pathol 2000;20:83-91.

32 Plancoulaine B, Laurinaviciene A, Herlin P, Besusparis J, Meskauskas R, Baltrusaityte I, Iqbal Y, Laurinavicius A: A methodology for comprehensive breast cancer Ki67 labeling index with intra-tumor heterogeneity appraisal based on hexagonal tiling of digital image analysis data. Virchows Arch 2015; 467:711-722.

33 Hanahan D, Weinberg RA: Hallmarks of cancer: the next generation. Cell 2011;144:646674.

34 Rakha EA, Ellis IO: Modern classification of breast cancer: should we stick with morphology or convert to molecular profile characteristics. Adv Anat Pathol 2011;18:255-267.

-35 Cuzick J, Dowsett M, Pineda S, Wale C, Salter J, Quinn E, Zabaglo L, Mallon E, Green AR, Ellis IO, Howell A, Buzdar AU, Forbes JF: Prognostic value of a combined estrogen receptor, progesterone receptor, Ki-67, and human epidermal growth factor receptor 2 immunohistochemical score and comparison with the genomic health recurrence score in early breast cancer. J Clin Oncol 2011;29: 4273-4278.

36 Barton S, Zabaglo L, A'Hern R, Turner N, Ferguson T, O’Neill S, Hills M, Smith I, Dowsett $\mathrm{M}$ : Assessment of the contribution of the IHC4+C score to decision making in clinical practice in early breast cancer. Br J Cancer 2012;106:1760-1765.
37 Afentakis M, Dowsett M, Sestak I, Salter J, Howell T, Buzdar A, Forbes J, Cuzick J: Immunohistochemical BAG1 expression improves the estimation of residual risk by IHC4 in postmenopausal patients treated with anastrazole or tamoxifen: a TransATAC study. Breast Cancer Res Treat 2013;140:253-262.

38 Rakha EA, Reis-Filho JS, Ellis IO: Combinatorial biomarker expression in breast cancer. Breast Cancer Res Treat 2010;120:293-308.

39 Laurinavicius A, Laurinaviciene A, Ostapenko V, Dasevicius D, Jarmalaite S, Lazutka J: Immunohistochemistry profiles of breast ductal carcinoma: factor analysis of digital image analysis data. Diagn Pathol 2012;7:27.

40 Laurinavicius A, Green AR, Laurinaviciene A, Smailyte G, Ostapenko V, Meskauskas R, Ellis IO: Ki67/SATB1 ratio is an independent prognostic factor of overall survival in patients with early hormone receptor-positive invasive ductal breast carcinoma. Oncotarget 2015;6:41134-41145

41 Joshi S, Watkins J, Gazinska P, Brown JP, Gillett CE, Grigoriadis A, Pinder SE: Digital imaging in the immunohistochemical evaluation of the proliferation markers Ki67, MCM2 and Geminin, in early breast cancer, and their putative prognostic value. BMC Cancer 2015; 15:546.

42 van den Brand M, Hoevenaars BM, Sigmans JH, Meijer JW, van Cleef PH, Groenen PJ, Hebeda KM, van Krieken JH: Sequential immunohistochemistry: a promising new tool for the pathology laboratory. Histopathology 2014;65:651-657.

43 Laurinaviciene A, Plancoulaine B, Baltrusaityte I, Meskauskas R, Besusparis J, LesciuteKrilaviciene D, Raudeliunas D, Iqbal Y, Herlin P, Laurinavicius A: Digital immunohistochemistry platform for the staining variation monitoring based on integration of image and statistical analyses with laboratory information system. Diagn Pathol 2014;9(suppl 1): S10. 